\title{
The center of pressure progression characterizes the dynamic function of high- arched feet during walking
}

\author{
Bo Li $i^{1}$ Qipeng Xiang ${ }^{1}$ and Xianyi Zhang ${ }^{1,2^{*}}$
}

\begin{abstract}
Background: The medial longitudinal arch height has an effect on kinetic parameters during gait and might be related to the risk of injury. For the assessment of foot structures, the center of pressure (COP) trajectory is a more reliable and practical parameter than plantar pressure. This study aimed to clarify the COP trajectory and velocity characteristics in the medial-lateral and anterior-posterior direction of individuals with a high-arched foot during barefoot walking.

Methods: Sixty-two healthy young adults were asked to walk over a Footscan pressure plate to record the COP parameters during the stance phase of walking.

Results: Compared to normal arched feet, the COP during forefoot contact and foot flat phases of high-arched feet shifted anteriorly $(19.9 \mathrm{~mm}$ and $15.1 \mathrm{~mm}$, respectively), and the mean velocity of COP in anterior-posterior direction decreased by $0.26 \mathrm{~m} / \mathrm{s}$ and increased by $0.044 \mathrm{~m} / \mathrm{s}$ during these two phases respectively.

Conclusions: The findings of this study suggest that the displacement and velocity of COP in anterior-posterior direction was different between high-arched and normal-arched subjects during barefoot walking, which can be used for the assessment of gait characteristics for high-arched individuals. The results of this study may provide insights into modifying clinical intervention for individuals with high-arched feet to enhance rehabilitation and prevent injuries and have implications for assessing the design of footwear and foot orthotics.
\end{abstract}

Keywords: High-arched, Walking, Center of pressure, Velocity

\section{Introduction}

Despite similarities in age and gender, foot dynamic function can differ considerably among individuals with different foot type. A high-arched foot is a foot type with an excessively high medial longitudinal arch (MLA). People with an extremely high MLA are more susceptible to injuries compared to individuals with a normal height of MLA [1]. Clinical management of high-arched feet with custom-made foot orthoses has been shown to be effective in some cases [2].

\footnotetext{
* Correspondence: xianyi.z@outlook.com

${ }^{1}$ Key Laboratory of Leather Chemistry and Engineering of Ministry of Education, Sichuan University, Chengdu, People's Republic of China ${ }^{2}$ Key Laboratory of Sensing Technology and Biomedical Instrument of Guangdong province, School of Biomedical Engineering, Higher Education Mega Center, Sun Yat-sen University, Building of Engineering College, East Campus, Panyu District, Guangzhou 510006, China
}

It has been suggested that mal-alignment and abnormal mechanics of the foot may contribute to lower extremity injuries. These injures are associated with the dynamic foot function in load attenuation and force transmission through the lower extremity kinetic chain $[3,4]$. Excessive plantar pressure under any foot zone may increase the risk of soft tissue injuries, which may result in pain [5]. A previous study reported a significant difference in plantar pressure distribution between individuals with normal and high-arched feet during walking [6]. It was indicated that the reduction of the weight-bearing contact area of individuals with high-arched feet might not lead to a pressure redistribution over all plantar zones. Instead, it only produced an overload on the anterior part of the foot.

The plantar pressure parameters, such as peak pressure and maximum force, can be used to investigate the loading underneath different plantar discrete region. 
However, identification of anatomical structures based on footprints is not very accurate [7], which is the base of plantar pressure distribution analysis. The masking protocol which determines the foot zones has a crucial effect on the plantar pressure variables, and thus may have an impact on the results [6]. The center of pressure (COP) is defined as the instantaneous point of application of the ground reaction forces. The COP moves predominantly in the heel-to-toe direction during the stance phase of walking [8], which represents the spatial distribution of plantar pressure over time [9]. It also portrays the spatial relationship between plantar pressure distribution and the entire plantar surface of the foot [10]. The COP analysis does not dependent on the accurate identification of anatomical structures from pressure-based footprints. For assessment of foot structure, the COP course is more reliable and practical than plantar pressure [11]. Displacement of the COP can provide insights into functional foot behavior and balance performance. Furthermore, the velocity of the COP provides information about foot loading pattern, gait pattern, and how gait changes, as the COP is associated with the whole body's center of mass during gait [12]. Therefore, it has been used as an important indicator in describing gait performance, such as movement efficiency, and stability control [13]. The displacement and velocity of COP are important indicators of the structure and dynamic function of the foot.

Mootanah et al. [14] assessed foot function using COP excursion index, which only provide information of the COP excursion at the distal one third of the foot in the medial-lateral direction. De Cock et al. [8] examined the COP trajectories of young adults during barefoot running and suggested that the characteristics of COP path could serve as reference for the dynamics of the foot rollover process and foot function. The COP displacement in the medial-lateral direction provided information on the MLA structure and dynamic functional behavior, while no effective parameters derived from the anterior-posterior direction of COP have been reported. Compared to running, walking is an activity with lower mechanical demand and smaller joint moments [4]. For young adults, there is a difference in temporal-spatial COP features in the sub-phases between walking and jogging. The different timing of the four sub-phases indicated different foot rollover temporal characteristics between walking and jogging $[11,15]$. De Cock et al. has outlined foot type and the displacement of COP in the medial-lateral direction during running, while the effect of foot type on the COP velocity has not been clearly defined. The dynamic behavior of COP during walking remains unclear. Buldt et al. compared the resultant velocity of COP characteristics between individuals with different foot types, including normal, planus and cavus feet [9]. They used a different foot type classification method, which was based on the foot posture index. This method consists of six validated, criterion-based observations of the forefoot and rearfoot of an individual standing in a relaxed position and is usually used in a variety of clinical settings [16]. This foot posture index method depends on trained and experienced raters, which may limit its use in footwear industry. While the foot type classification method using the arch index is based on a more objective measure, i.e. the footprint parameter characterized of a reduced midfoot contact area for high-arched feet, which is friendlier to footwear industry. Buldt et al. only reported the resultant velocity of COP, while the COP velocity in the medial-lateral and anterior-posterior dimensions may provide more information. Maintaining balance in the medial-lateral direction is critical during single support of walking [17], while dynamic stability is maintained mainly during the forefoot contact phase (FFCP) and the foot flat phase (FFP) of walking, corresponding to the function of weight-bearing stability and trunk stability, respectively [18]. Walking uses a repetitious sequence of limb motion to simultaneously move the body forward. The anteriorposterior progression of the body center of mass is reflected in the anterior-posterior COP progression during the stance phase of walking. The COP is a vector quantity with magnitude and direction characteristics [19]. Decomposing the COP into two components, such as anterior-posterior and medial-lateral components, may provide more meaningful information. In this study, the anterior-posterior component was defined with respect to the longitudinal foot axis, and the medial-lateral component was defined with perpendicular to the longitudinal foot axis. Thereby, the displacement and velocity of COP in the medial-lateral and anterior-posterior dimensions were analyzed and interpreted separately in the current study. It has been shown that the high arch structure exhibited different complex movement patterns, and thus different COP displacement and velocity, compared to normal arch structure [20]. Well-designed orthoses or footwear have been postulated to relieve foot pain and improve balance by restoring alignment, redistributing plantar load or limiting motion [21]. The displacement and velocity of the COP have been used as indicators for balance performance, plantar pressure characteristics and effectiveness of the orthoses or footwear $[8,10]$, which is essential to assess the gait characteristics for individuals with a high-arched foot during walking to predict the risk of foot pain or injury. Sample entropy has been used to quantify regularity and complexity of COP progression patterns [20, 22], which might be useful for recognizing foot abnormal deformation at an early stage. The displacement and velocity of COP have more explicit physical meanings, which is 
convenient for the structure design and effect examination of custom-made foot orthoses or footwear, compared to the sample entropy of COP velocity and acceleration.

The characteristics of COP trajectories in individuals with a high-arched foot may have implications for custom foot orthoses and footwear design for this foot type. Therefore, this study aimed to clarify the COP trajectory and velocity characteristics in the medial-lateral and anterior-posterior direction during barefoot walking in individuals with high-arched feet based on the footprint parameter (arch index) classification method. The effect of a high longitudinal arch on the COP progression was discussed through comparison with normal longitudinal arch using the displacement and velocity of COP, which provided a basis of clinical intervention for individuals with high-arched feet to reduce occurrence of foot pain. Due to a more medial COP course during running and a larger area for COP excursions during single-leg stance for the high-arched foot $[8,23]$, we hypothesized that the COP trajectory in individuals with high-arched feet was medially shifted and the stability was decreased during walking, compared to the normal-arched individuals.

\section{Materials and methods}

\subsection{Participants}

Participants were recruited from Sichuan University. Sixty-two healthy young adults (34 men and 28 women, 20-24 years old) gave informed consent and participated in this study. None of the participants had any lower extremity injuries in the last 6 months prior to testing. Arch indices between 0.21 and 0.26 were considered normal, and arch indices $\leq 0.21$ were considered higharched [24]. This determination was made by the arch index of right foot. The arch index was defined as the ratio of midfoot area divided by the total footprint area (excluding the toes), which was calculated by the Footscan analysis software based on the recorded dynamic pressure data. The calculation of the foot region contact area percentage was done according to the pressurebased footprints automatically.

The characterizations of sample are showed in Table 1 above. Subjects were similar in height, weight, body mass index (BMI), foot length and foot width for the higharched group and the control group. The data showed that the high-arched group had significantly higher arch index than the control group.

\subsection{Materials and apparatus}

A $1 \mathrm{~m}$ Footscan $^{\circ}$ pressure plate (RSscan International, Belgium, $1068 \times 418 \times 12 \mathrm{~mm}$, with 8192 resistive sensors arranged in a $128 \times 64$ matrix at a resolution of 2 sensors/ $\mathrm{cm}^{2}$, sensor dimensions: $5.08 \mathrm{~mm} \times 7.62 \mathrm{~mm}$, and pressure range: $0-127 \mathrm{~N} / \mathrm{cm}^{2}$ ) was used to measure the COP data with a measuring frequency of $250 \mathrm{~Hz}$. The medial-lateral COP displacement was calculated with respect to the longitudinal foot axis, which was defined as the line from midheel to mid-forefoot, between metatarsal head 2 and 3 .

\subsection{Procedures}

Participants were given time to get familiarized with the system and procedure. Then they walked along an $8-\mathrm{m}$ walkway with an integrated pressure plate at their preferred speed in barefoot condition. Five successful walking trials of each participant were recorded.

\subsection{Data analysis}

All analyses were performed on the right foot. According to the footscan ${ }^{\circ}$ analysis software, the stance phase was divided into four phases by dynamic pressure-based footprints. The initial contact phase (ICP) which is defined as the period from first foot contact until first metatarsal contact. The FFCP is the period from the first metatarsal contact until all metatarsal zones made contact with the pressure plate. The FFP follows the FFCP and ends when the heel is off the plate. The forefoot push-off phase (FFPOP) starts when the heel is lifted from the ground and ends when the foot is off the plate.

The mean COP trajectory of all trials for each participant were computed for further analysis and the figure of the trajectory can show the difference of the high-arched and the normal-arched feet visually. The COP displacement in the medial-lateral and anterior-posterior direction were normalized to each individual's foot width or foot length, respectively. To better illustrate the COP trajectories, foot length and width of all participants were averaged as the "standard foot". Then the mean displacements of the COP progression of all participants were plotted on the standard foot [11]. The medial-lateral and anteriorposterior velocity were calculated with the $\mathrm{x}$ - and $\mathrm{y}$ coordinates direction displacement divided by the elapsed

Table 1 Statistics of subjects

\begin{tabular}{llllllll}
\hline & Sex & Arch index & Height $(\mathrm{cm})$ & Weight $(\mathrm{kg})$ & BMl $\left(\mathrm{kg} / \mathrm{m}^{2}\right)$ & Foot length $(\mathrm{cm})$ & Foot width $(\mathrm{cm})$ \\
\hline High-arched $(n=31)$ & $14 \mathrm{~F}, 17 \mathrm{M}$ & $\mathbf{0 . 1 8} \pm \mathbf{0 . 0 2}$ & $167.6 \pm 8.2$ & $55.7 \pm 10.4$ & $19.5 \pm 2.3$ & $24.5 \pm 1.1$ & $8.7 \pm 0.6$ \\
Normal arch $(\mathrm{n}=31)$ & $20 \mathrm{~F}, 11 \mathrm{M}$ & $\mathbf{0 . 2 5} \pm \mathbf{0 . 0 1}$ & $168.6 \pm 3.8$ & $58.9 \pm 7.7$ & $20.7 \pm 2.4$ & $24.9 \pm 1.2$ & $8.5 \pm 0.3$ \\
$P$ value & & $\mathbf{0 . 0 0 0}$ & 0.591 & 0.215 & 0.085 & 0.208 & 0.312 \\
\hline
\end{tabular}

Bold values represent statistical difference with $p<0.05$ 
time between measurements respectively, and all trials were normalized for contact time. The mean, maximum and range for the displacement and velocity of COP were compared between two curves for more information. The range of the COP displacement and velocity of each stance phase was calculated as the absolute difference between the maximum and minimum $\mathrm{x}-, \mathrm{y}$-coordinate and velocity of the COP. The calculation was processed in the Matlab software (Math works Inc.).

Statistical analyses were performed using SPSS version 22 statistical analysis software. Each COP parameter was individually evaluated for normality, while all dependent variables that did not meet assumptions for normality were transformed. The differences between high-arched and normal-arched feet were assessed using independent samples t-test. If a measure did not achieve normality after transformation, a non-parametric test (Mann-Whitney Test) were performed to analyze the effect of the arch type on the COP variables. Significant differences between the two conditions were considered if $p<0.05$.

\section{Results}

There was no significant difference in the self-selected walking speed between two groups ( $p=0.271$ ), with $3.85 \pm$ $0.49 \mathrm{~km} / \mathrm{h}$ for high-arched group and $3.72 \pm 0.40 \mathrm{~km} / \mathrm{h}$ for control group.

The "standard foot" had foot length of $24.7 \pm 1.2 \mathrm{~cm}$ and foot width of $8.6 \pm 0.5 \mathrm{~cm}$. The trajectory of COP for the high-arched and the normal-arched feet are shown in Fig. 1. Figure 2 shows an example of the COP of the high-arched and the normal-arched feet.
The overall COP trajectory of the high-arched feet was medially shifted compared to the normal-arched feet. The main difference in mean COP displacement was observed during forefoot contact phase and foot flat phase. The y component of the COP showed a forward move during the whole stance phase. For the high-arched group, the $\mathrm{y}$-coordinates of $\mathrm{COP}$ at the initial contact point for foot flat phase was significantly increased, compared to the control group, which meant that the initial contact point of COP was displaced anteriorly for foot flat phase.

Comparison between the displacement and velocity of the medial-lateral COP for the high-arched group and the control group are shown in Table 2.

The mean displacement of ML-COP for high-arched was reduced by $1.5 \mathrm{~mm}(p=0.029)$ during the forefoot contact phase, the range displacement of ML-COP was reduced by $1.7 \mathrm{~mm}(p=0.018)$ during the foot flat phase, compared to the control group. There were no significant differences of the maximum of ML-COP between two conditions.

The maximum of the ML-COP velocity for the high-arched group reduced by $0.234 \mathrm{~m} / \mathrm{s} \quad(p=0.000)$ compared to the control group during the initial contact phase. The mean and maximum of the MLCOP velocity for the high-arched reduced by $0.051 \mathrm{~m} /$ $\mathrm{s} \quad(p=0.000)$ and $0.111 \mathrm{~m} / \mathrm{s} \quad(p=0.003)$ respectively during the forefoot contact phase, compared to the control group. The maximum and range of the MLCOP velocity for the high-arched group also reduced by $0.068 \mathrm{~m} / \mathrm{s} \quad(\mathrm{p}=0.000)$ and $0.048 \mathrm{~m} / \mathrm{s} \quad(p=0.001)$

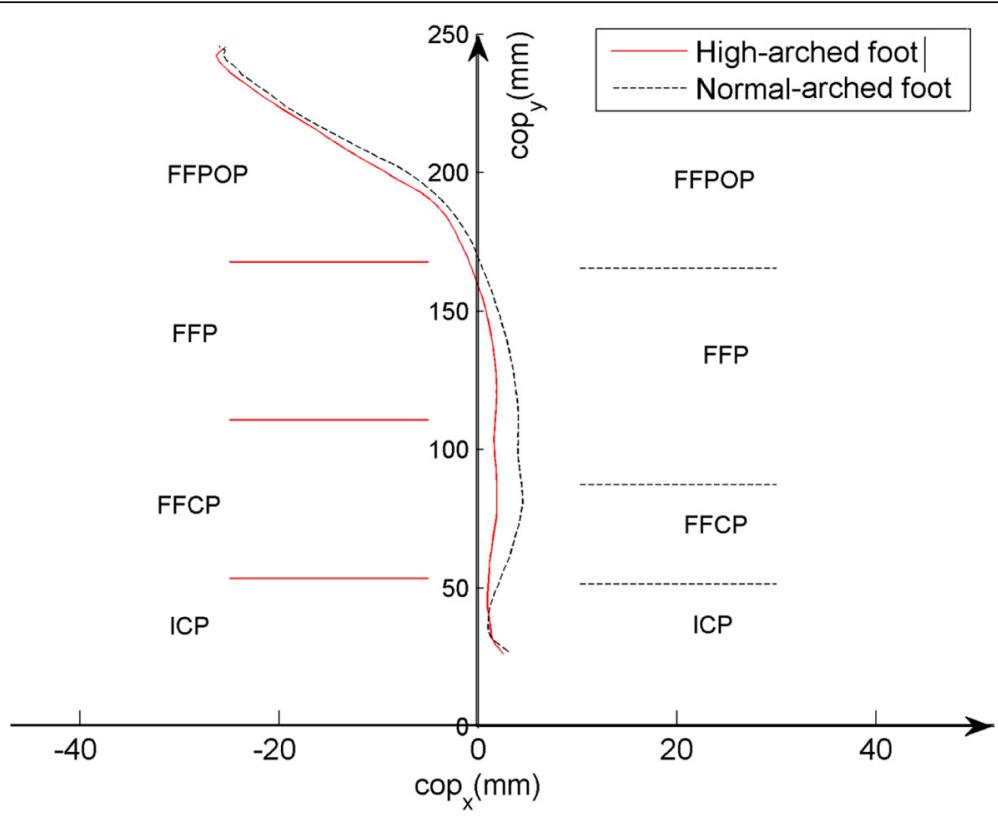

Fig. 1 The trajectory of COP of high-arched and normal-arched foot. The $y$-axis is the longitudinal axis of the foot, and $x$-axis is perpendicular to the longitudinal foot axis. Sub-classified phases are ICP, FFCP, FFP and FFPOP 

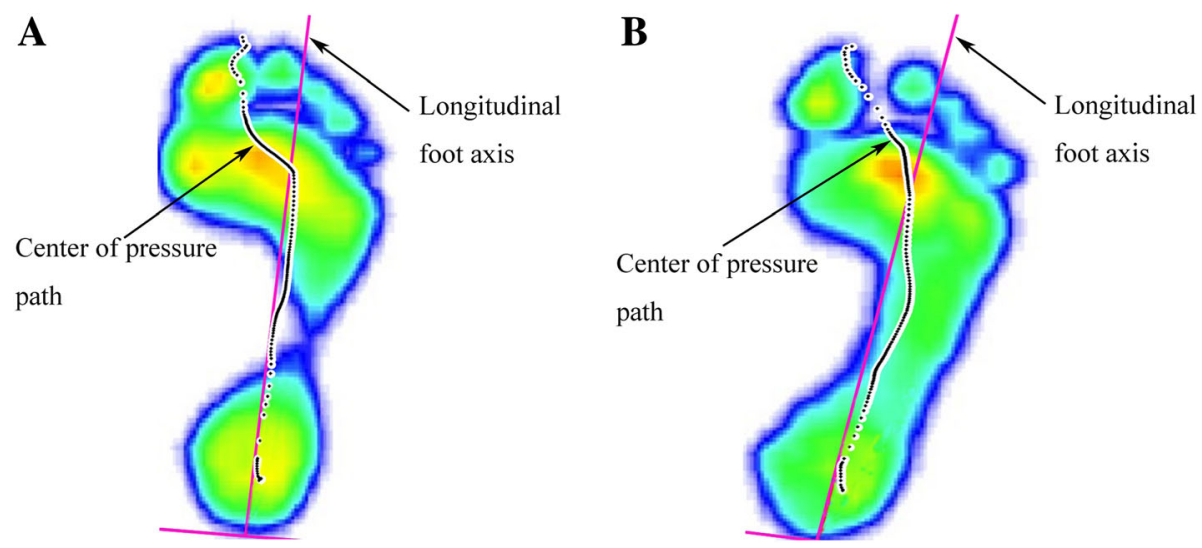

Fig. 2 Examples of COP of the high-arched (a) and normal-arched (b) feet

respectively during the foot flat phase, compared to the control group.

Comparison between the displacement and velocity of the anterior-posterior COP between these two groups are shown in Table 3.

The mean, maximum and range displacement of APCOP for high-arched group was increased by $19.9 \mathrm{~mm}$ $(p=0.000), 24.3 \mathrm{~mm}(p=0.000)$ and $25.4(\mathrm{p}=0.000)$ respectively during the forefoot contact phase, compared to the control group. The mean displacement of APCOP was increased by $15.1 \mathrm{~mm}(\mathrm{p}=0.000)$, the range displacement of AP-COP was reduced by $18.3 \mathrm{~mm}(p=$ 0.001) for high-arched group during the foot flat phase, compared to the control group. The range displacement of AP-COP for high-arched group was reduced by 7.6 $\mathrm{mm}(p=0.003)$ during the forefoot push off phase compared to the control group.

The mean of AP-COP velocity reduced by $0.265 \mathrm{~m} / \mathrm{s}$ $(p=0.011)$, the maximum and range of the ML-COP velocity increased by $0.813 \mathrm{~m} / \mathrm{s}(p=0.004)$ and $0.652 \mathrm{~m} / \mathrm{s}$ $(p=0.005)$ respectively for the high-arched group during the forefoot contact phase, compared to the control group. The mean of AP-COP velocity for the higharched group increased by $0.044 \mathrm{~m} / \mathrm{s}(p=0.048)$ during the foot flat phase, compared to the control group. The mean of AP-COP velocity reduced by $0.036 \mathrm{~m} / \mathrm{s}(p=$ 0.007 ), the maximum of the ML-COP velocity increased by $0.870 \mathrm{~m} / \mathrm{s}(p=0.011)$ for the high-arched group during the forefoot push off phase, compared to the control group. The velocity of COP in medial-lateral and anterior-posterior direction for the high-arched and the normal-arched feet are shown in Fig. 3.

\section{Discussion}

This study compared the COP variables during walking between high-arched and normal-arched individuals. Our results indicated that the trajectory of COP shifted medially in individuals with high-arched feet, especially

Table 2 Comparison of ML-COP deviation and velocity between high-arched and normal feet

\begin{tabular}{|c|c|c|c|c|c|c|c|}
\hline & & \multicolumn{3}{|c|}{ Deviation (mm) } & \multicolumn{3}{|l|}{ Velocity $(\mathrm{m} / \mathrm{s})$} \\
\hline & & High-arched & Normal Arch & $P$ value & High-arched & Normal arch & $P$ value \\
\hline \multirow[t]{4}{*}{ ML-COP mean } & ICP & $1.9(1.1)$ & $1.8(1.2)$ & 0.786 & $0.095(0.038)$ & $0.106(0.057)$ & 0.607 \\
\hline & FFCP & $3.0(1.7)$ & $4.5(2.8)$ & 0.029 & $0.039(0.022)$ & $0.090(0.058)$ & 0.000 \\
\hline & FFP & $4.3(2.5)$ & $4.8(2.8)$ & 0.687 & $0.026(0.015)$ & $0.028(0.010)$ & 0.221 \\
\hline & FFPOP & $11.1(5.5)$ & $10.8(4.5)$ & 0.998 & $0.107(0.041)$ & $0.117(0.029)$ & 0.137 \\
\hline \multirow[t]{4}{*}{ ML-COP max } & ICP & $3.5(1.4)$ & $3.9(2.2)$ & 0.361 & $0.235(0.123)$ & $0.469(0.303)$ & 0.000 \\
\hline & FFCP & $4.4(2.1)$ & $5.6(3.1)$ & 0.092 & $0.187(0.113)$ & $0.298(0.184)$ & 0.003 \\
\hline & FFP & $6.5(3.6)$ & $7.7(2.8)$ & 0.181 & $0.067(0.045)$ & $0.135(0.067)$ & 0.000 \\
\hline & FFPOP & $27.3(7.1)$ & $27.5(8.8)$ & 0.998 & $0.791(0.829)$ & $0.957(0.719)$ & 0.095 \\
\hline \multirow[t]{4}{*}{ ML-COP range } & ICP & $3.0(0.3)$ & $3.7(0.4)$ & 0.328 & $0.347(0.182)$ & $0.469(0.303)$ & 0.079 \\
\hline & FFCP & $3.5(0.4)$ & $3.6(0.3)$ & 0.569 & $0.259(0.176)$ & $0.298(0.184)$ & 0.302 \\
\hline & FFP & $4.4(0.6)$ & $6.1(0.5)$ & 0.018 & $0.087(0.070)$ & $0.135(0.067)$ & 0.001 \\
\hline & FFPOP & 26.1(1.1) & 28.0(1.3) & 0.240 & $0.994(1.198)$ & $0.957(0.719)$ & 0.495 \\
\hline
\end{tabular}


Table 3 Comparison of AP-COP deviation and velocity between high-archedes and normal feet

\begin{tabular}{|c|c|c|c|c|c|c|c|}
\hline & & \multicolumn{3}{|c|}{ Deviation (mm) } & \multicolumn{3}{|l|}{ Velocity $(\mathrm{m} / \mathrm{s})$} \\
\hline & & high-arched & normal arch & $P$ value & high-arched & normal arch & $P$ value \\
\hline \multirow[t]{4}{*}{ AP-COP mean } & ICP & $32.7(3.5)$ & $33.9(3.2)$ & 0.172 & $0.492(0.330)$ & $0.426(0.157)$ & 0.895 \\
\hline & FFCP & $90.5(14.6)$ & $70.6(13.4)$ & 0.000 & $0.458(0.164)$ & $0.723(0.405)$ & 0.011 \\
\hline & FFP & $135.4(16.1)$ & $120.3(9.4)$ & 0.000 & $0.336(0.089)$ & $0.292(0.087)$ & 0.048 \\
\hline & FFPOP & 199.1(5.8) & 198.1(5.7) & 0.496 & $0.241(0.053)$ & $0.277(0.050)$ & 0.007 \\
\hline \multirow[t]{4}{*}{ AP-COP max } & ICP & $44.6(9.3)$ & 46.3(8.9) & 0.454 & $1.387(1.168)$ & $1.168(1.138)$ & 0.848 \\
\hline & FFCP & 109.2(18.9) & $84.9(17.5)$ & 0.000 & $1.894(1.081)$ & $1.081(1.523)$ & 0.004 \\
\hline & FFP & 175.6(10.2) & 171.3(13.9) & 0.176 & $0.638(0.237)$ & $0.237(0.698)$ & 0.060 \\
\hline & FFPOP & 241.7(3.8) & 246.4(18.6) & 0.179 & $1.326(0.456)$ & $0.456(1.378)$ & 0.011 \\
\hline \multirow[t]{4}{*}{ AP-COP range } & ICP & 19.3(9.2) & 19.9(6.6) & 0.760 & $1.395(1.164)$ & $1.164(1.157)$ & 0.698 \\
\hline & FFCP & $59.6(16.8)$ & $34.2(10.8)$ & 0.000 & $1.811(1.159)$ & $1.159(1.272)$ & 0.005 \\
\hline & FFP & $65.8(22)$ & $84.1(19.5)$ & 0.001 & $0.513(0.316)$ & $0.316(0.629)$ & 0.217 \\
\hline & FFPOP & 64.9(9) & 72.5(10.4) & 0.003 & $1.319(0.604)$ & $0.604(1.368)$ & 0.765 \\
\hline
\end{tabular}

Bold values represent statistical difference with $p<0.05$

during forefoot contact and foot flat phase compared to the control group,which was in consistent with the trajectory of COP during running [8]. During initial contact and forefoot push off phase, the forefoot or rearfoot was not in contact with the ground respectively, which meant the shape or mobility of the arch cannot affect the absorption or transfer of forces significantly [25].

The COP deviation in the medial-lateral direction during sub-phases of walking is an important determinant for foot structure and dynamic function [11]. COP displacements in the medial-lateral direction mainly depend on the inversion-eversion movements of the foot, which are associated with the function of stability control, energy storage, and propulsion. Such movements are often performed by midfoot and subtalar joints in the frontal and transverse planes [26]. It has been suggested that even small distance changes (as little as $2 \mathrm{~mm}$ ) between the joint axis and the COP may alter the balance of moments acting across this joint axis, and may result in abnormal dynamic foot function and contributes to the development of biomechanical pathology [27, 28]. Although the group differences of COP displacement in the medial-lateral direction during the forefoot contact phase were statistically significant,the value of medial shift of COP path for high-arched feet compared to normal-arched feet was small. Taking the resolution of the Footscan pressure plate into consideration, the physiological meaning of this group difference in the medial-lateral COP displacement requires further investigation. The high longitudinal arch of the high-arched feet inhibited mid-foot contact, which caused the more medially located COP path in comparison to normalarched feet [8]. There was no significant differences of the range of ML-COP between two groups during forefoot contact phase of gait, which was different from the range of ML-COP during running [8]. According to the study of De Cock, a lower range of ML-COP motion for COP for high arched feet during running indicating the lesser flexibility of the foot [8], but the groups did not show difference in ML-COP during walking. This means that, in lower mechanical demand condition, the characteristic of lesser flexibility in high-arched feet was not observed, and that running may require more flexibility in the medial-lateral direction during forefoot contact phase than walking.

Smaller ML-COP velocity for high-arched feet during forefoot contact phase indicated a sluggish lateral weight shift compared to normal-arched feet. A possible explanation could be that the high longitudinal arch of higharched feet caused the lesser flexibility of the foot, which was not convenient to transfer load from lateral to medial side rapidly $[8,26]$. The finding was not in concordance with the observation by Mei et al. [20], in which there was no difference in the sample entropy of MLCOP velocity between the high-arched and normalarched feet group. Each stance period is consisted of several phases, which relates to a different function demand. The ML-COP velocity was assessed according to functional phase in this study, while no phases were divided in the study by Mei et al., which maybe was the reason of the disagreement between two studies.

The spatial progression of the COP along the longitudinal foot axis mainly depends on the articular mobility of the joints in the sagittal plane [26]. Interestingly, high-arched and normal-arched individuals adopted different paces during the four sub-stance phases. During forefoot contact phase, the mean, maximum and range of AP-COP for high-arched subjects was larger compared to normal-arched subjects,in other words, the COP for high-arched feet was anterior shifted. The forward 

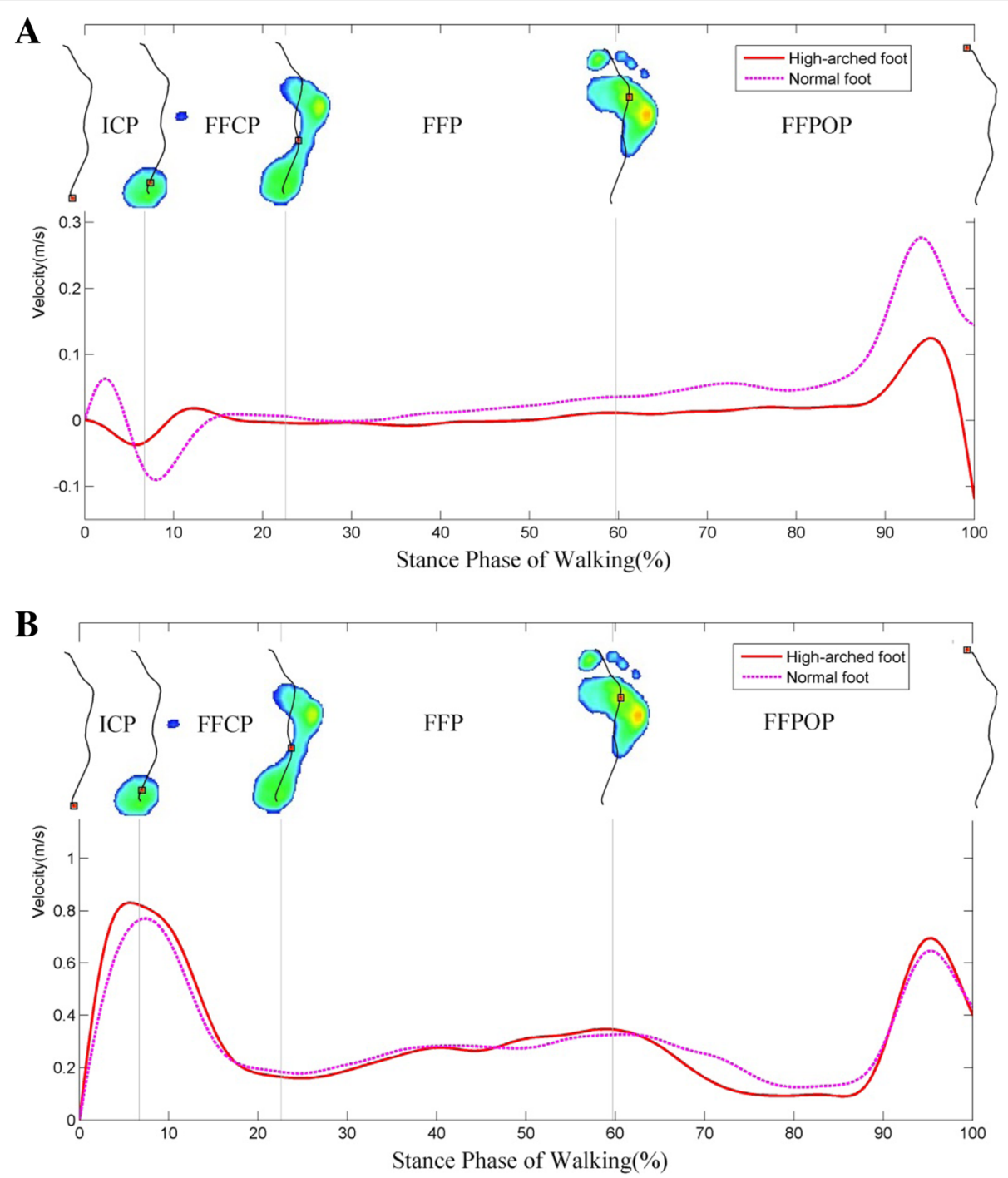

Fig. 3 The velocity of COP in medial-lateral (a) and anterior-posterior (b) direction for the high-arched and the normal-arched feet. The $y$-axis is the longitudinal axis of the foot, and $x$-axis is perpendicular to the longitudinal foot axis. Sub-classified phases are ICP, FFCP, FFP and FFPOP

movement process was a foot rollover and the anterior shifted COP for high-arched feet illustrated more forward movement, which might indicate the difference of foot rollover between these two foot types. This suggested comparatively greater force being borne on the anterior plantar surface of high-arched feet. The high longitudinal arch of high-arched feet caused the decreased contact area on midfoot, which limited the midfoot to distribute force, resulting in increased pressure on other areas. Increasing pressure coupled with an overload on forefoot might explain that the AP-COP showed an anterior shift during forefoot contact phase compared to normal-arched feet, which was indicative of the difference of plantar pressure distribution by shifting part of the load from the posterior side of the foot to the anterior side [6]. In this situation, an arch support can reduce plantar pressure [29]. Peak forces of the forefoot could be reduced as pressure was dispersed across the greater contact surfaces that an arch support provided. The AP-COP value for high-arched subjects may be reduced as the force was shifted to the midfoot during forefoot contact phase.

The forward rolling of the foot depends on a moment, rather than a force alone. The effect of COP location relative to a joint axis can be used to learn the moment from ground reactive force acting on the joint. Forward progression during walking is produced primarily by the ankle plantar flexor muscles [12]. During forefoot contact phase, the ankle joint is planter flexed. The sagittal anterior shifted COP extended the distance between the ground reaction force and the center of the ankle joint, resulting in increased magnitude of the torques [30]. 
Therefore, the anterior shifted COP for high-arched feet increased the rocker moment arm and consequently increased the demand of muscular control during this phase.

The trajectory of COP contains information about its progression patterns [26]. The COP progression may reflect a movement strategy aiming at improving stability and adaptability, which can be caused by the postural adjustments in the hip and knee when the body attempts to establish stability [31]. Rapid ankle plantar flexion with fast initial pronation occurred during forefoot contact phase, AP-COP velocity peaks indicated a rapid forward weight shift $[8,32]$. A slower mean AP-COP velocity of the high-arched foot during forefoot contact phase indicated a sluggish forward weight shift, while the maximum and range of AP-COP velocity of the high-arched foot was faster compared to normal-arched individuals. This indicated that the regularity of APCOP velocity for individuals with high-arched feet was worse compared to normal-arched individuals, which accompanied with an abnormal fold-back of the COP under the arch area in the anterior-posterior direction sometimes. The result was in consistent with the finding of Mei et al. [20]. The additional directional changes in the anterior-posterior COP trajectory might indicate that individuals with high-arched feet were less stable [33]. This might be because the high-arched foot has less plantar sensory information to rely on compared to the normal-arched foot, which results in decreased stability [34]. The result was in consistent with the finding by Hertel et al. [23]. An arch is the most pressure sensitive region of the foot. Adding an arch support to custom foot orthoses or footwear can improve the stability for individuals with high-arched feet during walking [35].

According to our previous study, wearing a heel lift or a high-heeled shoe may produce a foot alignment that resemble a high-arched foot in individuals with normal arches [29]. A heel lift or a high-heeled shoe can change the foot alignment by lifting the midfoot and rearfoot away from the ground. The midfoot could be raised further due to the windlass mechanism of plantar aponeurosis in such a foot alignment. Therefore, both medial and lateral longitudinal arches might be suspended and the midfoot contact area might be reduced due to the use of inappropriate heel lifts and high-heeled shoes. The reason for the situation mentioned above was wearing a poorly designed heel lift or high-heeled shoe. A heel lift or high-heeled shoe generated biomechanical manipulations may alter the COP trajectory,thereby altering the location of the ground reactive force and modifying moments and forces acting on the proximal body segments [36]. According to the contour of the plantar, the shoe-foot interface shape or insole contour can be altered, thus the COP can be changed. This may provide implications for optimizing the design of a heel lift or high-heeled shoe. The evolution of the design of a heel lift or high-heeled can benefit from biomechanical analysis. For instance, the AP-COP parameters can be used as feedback when optimizing the design of a heel lift or high-heeled shoe. Alterations in the design is required until the heel lift or high-heeled shoe provides an optimal AP-COP during dynamic movements.

The fundamental objectives of the optimize design of a heel lift or high-heeled are reducing the plantar load and improving performance. A well-designed arch support can not only reduce peak force of the forefoot but also improve stability control $[29,35,37]$. The AP-COP displacements and the regularity of AP-COP velocity during forefoot contact phase can be used to optimize and assess the design of the heel lift or high-heeled shoe.

The foot flat phase is the period when the body weight transfers from the rearfoot to the forefoot, with the ankle joint dorsiflexed to rise the rearfoot, and the subtalar joint locked to increase the stiffness of the foot for preparation of propulsion [32]. During the foot flat phase, the body weight continuously passes over the foot and the foot changes from a flexible to a rigid structure. Our results suggested a smaller ML-COP motion range, maximum and range of ML-COP velocity for higharched feet during foot flat phase compared to normalarched feet, which probably indicated the high stiffness of the high-arched feet, indicating that the high-arched feet was easy to change from a flexible to a rigid structure. A similar situation was found during forefoot contact phase of running with smaller range of motion of ML-COP as high-arched athletes had smaller range of ankle motion in the frontal plane [8] .

The COP velocity during foot flat phase described the functional ankle rocker characteristics. The faster mean AP-COP velocity for high-arched feet during foot flat phase compared to control group indicated that the velocity of body weight passing over the foot was faster, which corresponded to the rigid structure of higharched feet and guaranteed the efficient progression of the body. The increase velocity tended to produce a relatively faster body weight shift from the rear foot to the forefoot, which may be the reason of overload on the forefoot for individuals with high-arched feet [6] . Cushion in forefoot has the effect on delay time of forefoot contact or decrease the COP velocity in the foot flat phase during walking, which may figure out the overload on the forefoot for individuals with high-arched feet.

The displacement of AP-COP can be used as indicator of plantar load distribution in the longitudinal foot axis direction and the velocity of AP-COP can be used to evaluate the foot rollover function. Therefore, investigating the characteristics of the AP-COP is a research tool for the dynamic function of high-arched feet and 
effectiveness of heel lift or high-heel shoe. The main novelty of the proposed method is taking into account dynamic characteristics of AP-COP progression for high-arched feet during walking.

One limitation arising from the current study should be noted. The participants of this study were young healthy subjects. As such, the results of the study are valid only for subjects with characteristics similar to this group. As age and BMI have an influence on the COP trajectory [32, 38], Different populations e.g. the elderly and obesity may demonstrate different COP characteristics. Further studies are needed before these findings can be extended to other populations.

\section{Conclusion}

The detailed comparison of the COP variables between high-arched and normal-arched feet suggests that the displacement and velocity of COP in anterior-posterior direction can be used for the assessment of gait characteristics during walking. The characteristics of the COP trajectory were different between high-arched and normal-arched subjects during barefoot walking. Treatment for individuals with high-arched can be directed with custom-made foot orthoses or footwear at observing of the COP and making alteration with an arch support and cushion in forefoot. The results of this study may provide insights into modifying clinical intervention for individuals with high-arched feet to enhance rehabilitation and prevent injuries and have implications for assessing the design of footwear and foot orthotics.

\section{Abbreviations}

BMI: Body mass index; COP: Center of pressure; FFCP: Forefoot contact phase; FFP: Foot flat phase; FFPOP: Forefoot push-off phase; ICP: Initial contact phase; MLA: Medial longitudinal arch

\section{Acknowledgements}

The authors would like to thank Yingxu Zhong and Jiahua Zhao for their help on tidying up the data.

\section{Authors' contributions}

QX contributed to the data collection and data analysis. XZ contributed to the study design and manuscript preparation. BL contributed to the study design and manuscript preparation. All authors read and approved the final manuscript.

\section{Authors' information}

$\mathrm{BL}$ is mainly engaged in the teaching and research of foot biomechanics in Sichuan University.

QX is a graduate student in Sichuan University.

$\mathrm{XZ}$ is a PhD graduated from the University of Leuven in Belgium.

\section{Funding}

Not applicable.

\section{Availability of data and materials}

All data generated or analyzed during this study are included in this published article.

\section{Competing interests}

The authors declare that they have no competing interests.
Received: 20 May 2019 Accepted: 14 November 2019

Published online: 06 January 2020

\section{References}

1. Kaufman K, Brodine S, Shaffer R, Johnson C, Cullison T. The effect of foot structure and range of motion on musculoskeletal overuse injuries. Am J Sports Med. 1999;27(5):585-93.

2. Zifchock RA, Davis I. A comparison of semi-custom and custom foot orthotic devices in high- and low-arched individuals during walking. Clin Biomech. 2008;23(10):1287-93.

3. Chang A, Hurwitz D, Dunlop D, Song J, Cahue S, Hayes K, Sharma L. The relationship between toe-out angle during gait and progression of medial tibiofemoral osteoarthritis. Ann Rheum Dis. 2007:66(10):1271-5.

4. Powll DW, Williams DSB, Windsor B. Ankle work and dynamic joint stiffness in high- compared to low-arched athletes during a barefoot running task. Hum Mov Sci. 2014;34:147-56.

5. Nakhaee Z, Rahimi A, Abaee M, Rezasoltani A, Kalantari KK. The relationship between the height of the medial longitudinal arch (MLA) and the ankle and knee injuries in professional runners. Foot (Edinb). 2008;18(2):84-90.

6. Fernandez-Seguin LM, Diaz Mancha JA, Sanchez Rodriguez R, Escamilla Martinez E, Gomez Martin B, Ramos OJ. Comparison of plantar pressures and contact area between normal and cavus foot. Gait Posture. 2014;39(2): 789-92.

7. Deschamps K, Birch I, Mc Innes J, Desloovere K, Matricali GA. Inter- and intra-observer reliability of masking in plantar pressure measurement analysis. Gait Posture. 2009;30(3):379-82.

8. De Cock A, Vanrenterghem J, Willems T, Witvrouw E, De Clercq D. The trajectory of the Centre of pressure during barefoot running as a potential measure for foot function. Gait Posture. 2008;27(4):669-75.

9. Buldt AK, Forghany S, Landorf KB, Murley GS, Levinger P, Menz HB. Centre of pressure characteristics in normal, planus and cavus feet. J Foot Ankle Res. 2018;11(1):3.

10. Wong L, Hunt A, Burns J, Crosbie J. Effect of foot morphology on center-ofpressure excursion during barefoot walking. J Am Podiatr Med Assoc. 2008; 98(2):112-7.

11. Chiu MC, Wu HC, Chang LY. Gait speed and gender effects on center of pressure progression during normal walking. Gait Posture. 2013:37(1):43-8.

12. Fuchioka S, Iwata A, Higuchi Y, Miyake M, Kanda S, Nishiyama T. The forward velocity of the Center of Pressure in the Midfoot is a major predictor of gait speed in older adults. Int J Gerontol. 2015;9(2):119-22.

13. Cornwall MW, Mcpoil TG. Velocity of the center of pressure during walking. J Am Podiatr Med Assoc. 2000;90(7):334-8.

14. Mootanah R, Song J, Lenhoff MW, Hafer JF, Backus SI, Gagnon D, Deland JT 3rd, Hillstrom HJ. Foot type biomechanics part 2: are structure and anthropometrics related to function? Gait Posture. 2013;37(3):452-6.

15. De Cock A, De Clercq D, Willems T, Witvrouw E. Temporal characteristics of foot roll-over during barefoot jogging: reference data for young adults. Gait Posture. 2005:21(4):432-9.

16. Redmond AC, Crane YZ, Menz HB. Normative values for the foot posture index. J Foot Ankle Res. 2008;1(1):6.

17. Kanaya C, Takabayashi T, Inai T, Kubo M. Evaluation of one-foot standing using medial-lateral/anterior-posterior ratio of mean COP velocity. Physiotherapy. 2015;101:714-5.

18. Zhang $X$, Li B. Influence of in-shoe heel lifts on plantar pressure and center of pressure in the medial-lateral direction during walking. Gait Posture. 2014;39(4):1012-6.

19. Rhea CK, Kiefer AW, Haran FJ, Glass SM, Warren WH. A new measure of the CoP trajectory in postural sway: dynamics of heading change. Med Eng Phys. 2014;36(11):1473-9.

20. Zhao G, Zhu Q, Zhou Y, Wang L, Mei. Sample entropy characteristics of movement for four foot types based on;plantar centre of pressure during stance phase. Biomed Eng Online. 2013;12(1):101.

21. Rao S, Riskowski JL, Hannan MT. Musculoskeletal conditions of the foot and ankle: assessments and treatment options. Best Pract Res Clin Rheumatol. 2012;26(3):345-68

22. Mei Z, Ivanov K, Zhao G, Li H, Wang L. An explorative investigation of functional differences in plantar center of pressure of four foot types using sample entropy method. Med Biol Eng Comput. 2017:55(4):537-48.

23. Hertel J, Gay MR, Denegar CR. Differences in postural control during singleleg stance among healthy individuals with different foot types. J Athl Train. 2002;37(2):129-32 
24. Cavanagh PR, Rodgers MM. The arch index: a useful measure from footprints. J Biomech. 1987;20(5):547-51.

25. Williams DS 3rd, Tierney RN, Butler RJ. Increased medial longitudinal arch mobility, lower extremity kinematics, and ground reaction forces in higharched runners. J Athl Train. 2014;49(3):290-6.

26. Pan X, Bai J-J, Sun J, Ming Y, Chen L-R, Wang Z. The characteristics of walking strategy in elderly patients with type 2 diabetes. Int J Nurs Sci. 2016:3(2):185-9.

27. Kirby KA. Biomechanics of the normal and abnormal foot. J Am Podiatr Med Assoc. 2000;90(1):1-5.

28. Shelburne KB, Torry MR, Steadman JR, Pandy MG. Effects of foot orthoses and valgus bracing on the knee adduction moment and medial joint load during gait. Clin Biomech. 2008;23(6):814-21.

29. Zhang X, Li B, Liang K, Wan Q, Vanwanseele B. An optimized design of inshoe heel lifts reduces plantar pressure of healthy males. Gait Posture. 2016:47:43-7.

30. Haim A, Rozen N, Wolf A. The influence of sagittal center of pressure offset on gait kinematics and kinetics. J Biomech. 2010;43(5):969-77.

31. Luximon Y, Cong Y, Luximon A, Zhang M. Effects of heel base size, walking speed, and slope angle on center of pressure trajectory and plantar pressure when wearing high-heeled shoes. Hum Mov Sci. 2015;41:307-19.

32. Chiu MC, Wu HC, Chang LY, Wu MH. Center of pressure progression characteristics under the plantar region for elderly adults. Gait Posture. 2013; 37(3):408-12

33. Sinitski EH, Herbert-Copley AG, Lemaire ED, Doyle SS, Besemann M, Dudek NL. Center of pressure and total force analyses for amputees walking with a backpack load over four surfaces. Appl Ergon. 2016;52:169-76.

34. Cote KP, Michael E, Brunet ME II, Gansnede BM. Effects of pronated and supinated foot postures on static and dynamic postural stability. J Ath Train. 2005;40(1):41-6.

35. Zhang X, Li B, Hu K, Wan Q, Ding Y, Vanwanseele B. Adding an arch support to a heel lift improves stability and comfort during gait. Gait Posture. 2017:58:94-7.

36. Mona K, Alon W, Debbi EM, Amir H, Amir H. Foot center of pressure trajectory alteration by biomechanical manipulation of shoe design. Foot Ankle Int. 2013;34(4):593-8.

37. Debbie M, Taggart HM, Anita N, Camille P. Arch support use for improving balance and reducing pain in older adults. Appl Nurs Res. 2008;21(3):153-8.

38. Melzer I, Oddsson LI. Altered characteristics of balance control in obese older adults. Obes Res Clin Pract. 2016;10(2):151-8.

\section{Publisher's Note}

Springer Nature remains neutral with regard to jurisdictional claims in published maps and institutional affiliations.

\section{Submit your manuscript to a SpringerOpen ${ }^{\circ}$ journal and benefit from:}

- Convenient online submission

- Rigorous peer review

- Open access: articles freely available online

High visibility within the field

- Retaining the copyright to your article

Submit your next manuscript at $\boldsymbol{\nabla}$ springeropen.com 